\title{
Mycobacterium marseillense sp. nov., Mycobacterium timonense sp. nov. and Mycobacterium bouchedurhonense sp. nov., members of the Mycobacterium avium complex
}

\author{
Iskandar Ben Salah, Caroline Cayrou, Didier Raoult and Michel Drancourt
}

Correspondence

Michel Drancourt

Michel.Drancourt@univmed.fr
Unité de Recherche sur les Maladies Infectieuses et Tropicales Emergentes, UMR, CNRS-IRD 6236, IFR 48 Faculté de Médecine, Université de la Méditerranée, Marseille, France

\begin{abstract}
An rpoB sequence-based evaluation of 100 Mycobacterium avium complex (MAC) clinical isolates led to the identification of five respiratory tract isolates that were potential representatives of three novel MAC species. Distinctive phenotypic features of isolates 62863 and $5356591^{\top}$ included a pseudomycelium morphology and both esterase and acid phosphatase activities. These two isolates exhibited sequence similarities of $99.8 \%$ for the $16 \mathrm{~S}$ rRNA gene, 86.3 and $86.1 \%$ for $16 \mathrm{~S}-23 \mathrm{~S}$ rRNA gene internal transcribed spacer (ITS-1) sequence, 96.7 and $97.8 \%$ for $r p o B$ and 97.6 and $97.4 \%$ for $h s p 65$, respectively, with the type strain of Mycobacterium chimaera, the most closely related species. Isolates 3256799 and $5351974^{\top}$ lacked $\alpha-$ mannosidase and $\beta$-glucosidase activities. They exhibited sequence similarities of $99.6 \%$ for the 16S rRNA gene, 90.1 and $90.4 \%$ for ITS-1, $97.8 \%$ for $r p o B$ and 98.0 and $98.1 \%$ for $h s p 65$, respectively, with the type strain of $M$. chimaera, the most closely related species. Isolate $4355387^{\top}$ lacked urease and $\alpha$-glucosidase activities, but it exhibited valine arylamidase, cystine arylamidase and acid phosphatase activities. It had sequence similarities of $99.3 \%$ for the $16 \mathrm{~S}$ rRNA gene, $51.8 \%$ for ITS-1, $97.1 \%$ for $r p o B$ and $97.8 \%$ for $h s p 65$ with the type strain of Mycobacterium colombiense, the most closely related species. A phylogenetic tree based on concatenated 16S rRNA gene, ITS-1, rpoB and hsp65 sequences showed the uniqueness of these five isolates as representatives of three novel species, with bootstrap values $\geqslant 95 \%$ in all nodes. On the basis of these phenotypic and genetic characteristics, these five isolates are proposed as representatives of three novel MAC species: Mycobacterium marseillense sp. nov., with strain $5356591^{\top}\left(=\right.$ CCUG $56325^{\top}=$ CIP $109828^{\top}=$ CSUR P30 $\left.{ }^{\top}\right)$ as the type strain; Mycobacterium timonense sp. nov., with strain $5351974^{\top}$ (= CCUG $56329^{\top}={\text { CIP } 109830^{\top}}^{\top}$ $=$ CSUR P $32^{\top}$ ) as the type strain; and Mycobacterium bouchedurhonense sp. nov., with strain $4355387^{\top}\left(=\right.$ CCUG $56331^{\top}=$ CIP $109827^{\top}=$ CSUR P34 $\left.4^{\top}\right)$ as the type strain.
\end{abstract}

The Mycobacterium avium complex (MAC) initially comprised two species, namely Mycobacterium avium and Mycobacterium intracellulare (Pfyffer, 2007). However, recent studies have drawn attention to the wide diversity of isolates that can be detected in this complex (Lebrun et al., 2005; Mijs et al., 2002). Analysis of the 16S-23S rRNA gene internal transcribed spacer (ITS-1) sequence, a molecular approach used frequently for the identification of these mycobacteria, has revealed the existence of more than 30 different sequevars (Tortoli et al., 2004). Only a

Abbreviations: ITS, internal transcribed spacer; MAC, Mycobacterium avium complex.

The GenBank/EMBL/DDBJ accession numbers for the 16S rRNA gene, rpoB, hsp 65 and ITS- 1 sequences of the novel isolates are given in Tables 2 and 3. few of these sequevars have been assigned to $M$. avium (seven sequevars) and $M$. intracellulare (four sequevars). Recently, sequevar MAC-A organisms were assigned to a novel species, Mycobacterium chimaera (Tortoli et al., 2004). Likewise, MAC-X organisms were gathered in a fourth species, Mycobacterium colombiense (Murcia et al., 2006). We recently introduced partial $r p o B$ sequencing for the molecular identification of MAC organisms at the species level and showed that a $99.3 \%$ rpoB sequence similarity cut-off could be used to delineate MAC species (Ben Salah et al., 2008). When applying $r p o B$ sequencing to a collection of clinical MAC isolates cultured in our laboratory, five isolates were found whose partial $r p o B$ sequence similarity with the four MAC species was below the $99.3 \%$ cut-off. We further characterized these five isolates by using a polyphasic approach and herein present 
phenotypic and genetic evidence for these isolates being representatives of three novel MAC species.

\section{Mycobacterium isolates}

Strains 62863 and $5356591^{\mathrm{T}}$ were recovered from the bronchial aspirate and sputum, respectively, of two unrelated patients. Strains $3256799,5351974^{\mathrm{T}}$ and $4355387^{\mathrm{T}}$ were recovered from sputum specimens of three other unrelated patients. All clinical specimens were inoculated into BACTEC $9000 \mathrm{MB}$ broth after standard decontamination, according to the manufacturer's instructions (Beckton Dickinson Biosciences). The five clinical isolates, as well as seven MAC reference strains, M. avium subsp. avium ATCC $25291^{\mathrm{T}}$, M. avium subsp. silvaticum ATCC $49884^{\mathrm{T}}$, M. avium subsp. paratuberculosis ATCC $19698^{\mathrm{T}}$, 'M. avium subsp. hominissuis' IWGMT 49 (IWGMT: International Working Group on Mycobacterial Taxonomy), a kind gift of $\mathrm{D}$. van Soolingen (National Mycobacteria Reference Laboratory, Bilthoven, The Netherlands), $M$. intracellulare ATCC 15985, M. chimaera DSM $44623^{\mathrm{T}}$ and M. colombiense CIP $108962^{\mathrm{T}}$, were subcultured on Middlebrook 7H10 agar.

\section{Morphological features and cultivation}

Initially, colony morphology, pigmentation and the ability of the isolates to grow at $25,30,37$ and $45{ }^{\circ} \mathrm{C}$ were observed. Cultures were grown in the presence of $5 \% \mathrm{NaCl}$ on Middlebrook 7H10 agar, egg-based Löwenstein-Jensen slants (bioMérieux) and $5 \%$ sheep blood agar (BioTechnologie Appliqué) at $37{ }^{\circ} \mathrm{C}$ under a $5 \% \mathrm{CO}_{2}$ atmosphere. Subculture of the five clinical isolates on Middlebrook 7H10 agar yielded rough and non-pigmented colonies after 10 days of incubation at $30{ }^{\circ} \mathrm{C}$. Neither clinical isolates nor MAC reference strains grew on MacConkey agar or Löwenstein-Jensen agar supplemented with $5 \% \mathrm{NaCl}$ after 10 days of incubation at $37{ }^{\circ} \mathrm{C}$. Strains $3256799,5351974^{\mathrm{T}}$ and $4355387^{\mathrm{T}}$, as well as reference strains of $M$. avium subsp. avium, M. avium subsp. silvaticum, ' $M$. avium subsp. hominissuis' and $M$. intracellulare, grew on Middlebrook $7 \mathrm{H} 10$ agar at $45{ }^{\circ} \mathrm{C}$. All isolates were positive for Gram staining and they appeared as acid-fast bacilli following Ziehl-Neelsen staining.

Samples were prepared for electron microscopy as follows. A drop of mycobacterial suspension in PBS was deposited on a piece of Parafilm, which was placed on a 400-mesh Formvar carbon-coated copper grid and incubated for $15 \mathrm{~min}$ at $37^{\circ} \mathrm{C}$. The grid was then dried using filter paper (Macherey Nagel) and placed in $2 \%$ phosphotungstic acid for $10 \mathrm{~s}$. The grid was dried again and deposited on the filter paper with the Formvar facing upwards. This grid was observed using a Morgagni M 268D electron microscope $(\mathrm{FEI})$ at $60 \mathrm{kV}$ with $\times 28000$ magnification. Images were analysed using ANALYSIS PRO3.2 software. Electron microscopy observations indicated that all isolates looked like bacilli, with pseudomycelium formations apparent in strains $5356591^{\mathrm{T}}$ and 62863 . Cells of all five clinical isolates were generally shorter than those of the MAC reference strains (Table 1).

\section{Biochemical tests}

Urease activity and the ability to take up iron were tested as described previously (Kent \& Kubica, 1985; Vincent et al., 2003). Additional biochemical tests were performed by inoculation of API Coryne, API 20E and API ZYM strips (bioMérieux) for 5 days at $30{ }^{\circ} \mathrm{C}$ under a highly humidified atmosphere (Table 1). With the exception of strain $5356591^{\mathrm{T}}$, all isolates were negative in the niacin test and were unable to hydrolyse Tween 80 and were positive for iron fixation and potassium tellurite reduction. Strains $5356591^{\mathrm{T}}$ and 62863 differed from M. chimaera DSM $44623^{\mathrm{T}}$ in their positive esterase and acid phosphatase activities. Strains 3256799 and $5351974^{\mathrm{T}}$ differed from $M$. chimaera DSM $44623^{\mathrm{T}}$ in their lack of $\alpha$-mannosidase and $\beta$-glucosidase activities. Strain $4355387^{\mathrm{T}}$ differed from $M$. colombiense CIP $108962^{\mathrm{T}}$ by its lack of urease and $\alpha$ glucosidase activities and the presence of cystine arylamidase, valine arylamidase and acid phosphatase activities, as well as use of citrate as a sole carbon source (Table 1).

\section{Genotypic characterization}

After inactivation (Djelouagji \& Drancourt, 2006), genomic DNA was extracted from cells using a QIAamp DNA Mini kit (Qiagen). PCR amplification and sequencing were performed for the 16S rRNA gene (Weisburg et al., 1991), the ITS-1 spacer (Frothingham \& Wilson, 1993) and for parts of the hsp65 (Turenne et al., 2006) and rpoB genes (Ben Salah et al., 2008). Products of the sequencing reactions were analysed with an ABI Prism 3100 DNA sequencer following the standard protocol of the supplier (Perkin Elmer Applied Biosystems). Accession numbers for the sequences of the $16 \mathrm{~S}$ rRNA gene, $r p o B$, $h s p 65$ and ITS- 1 are given in Tables 2 and 3. Similarities (\%) between the sequences were determined by BIOEDIT (http:// www.mbio.ncsu.edu/BioEdit/bioedit.html) using the CLUSTAL W program supported by the PBIL website (http:// npsa-pbil.ibcp.fr/; Tables 2 and 3). Sequences were trimmed to start and finish at the same nucleotide position for all of the isolates. Multiple sequence alignment was performed by use of the program CLUSTAL_X version 1.81 in the PHYLIP software package (Thompson et al., 1997). Using Mycobacterium tuberculosis $\mathrm{H}_{3} 7 \mathrm{Rv}^{\mathrm{T}}$ as an outgroup, a phylogenetic tree was obtained from DNA sequences using the neighbour-joining method with Kimura's two-parameter distance correction model with 1000 bootstrap replications in the MEGA version 3.1 software package (Kumar et al., 2004).

The 16S rRNA gene sequence, which is used widely for identification and phylogenetic analysis of novel bacterial species, is not informative for Mycobacterium species (Mignard \& Flandrois, 2008). In the MAC, for example, the two species $M$. intracellulare and M. chimaera differ by 
Table 1. Distinctive biochemical characteristics of MAC isolates and reference strains

Strains: 1, strain $5356591^{\mathrm{T}}$; 2, strain 62863; 3, strain 3256799; 4, strain $5351974^{\mathrm{T}}$; 5, strain $4355387^{\mathrm{T}}$; 6, M. avium subsp. avium ATCC 25291 ${ }^{\mathrm{T}}$; 7 ' 'M. avium subsp. hominissuis' IWGMT 49; 8, M. avium subsp. paratuberculosis ATCC $19698^{\mathrm{T}}$; 9, M. avium subsp. silvaticum ATCC 49884 ${ }^{\mathrm{T}}$; 10 , M. intracellulare ATCC 15985; 11, M. chimaera DSM 44623 ${ }^{\mathrm{T}}$; 12, M. colombiense CIP $108962^{\mathrm{T}}$. +, Positive; -, negative; +/-, weakly positive.

\begin{tabular}{|c|c|c|c|c|c|c|c|c|c|c|c|c|}
\hline Characteristic & 1 & 2 & 3 & 4 & 5 & 6 & 7 & 8 & 9 & 10 & 11 & 12 \\
\hline \multicolumn{13}{|l|}{ Cell size $(\mu \mathrm{m})$} \\
\hline Length & 1.03 & 0.92 & 0.90 & 0.90 & 0.87 & 0.97 & 1.28 & 1.45 & 1.88 & 1.52 & 1.54 & 1.39 \\
\hline Growth at $45{ }^{\circ} \mathrm{C}$ & + & + & + & + & + & + & + & - & + & + & - & - \\
\hline Pigmentation & - & - & - & - & - & - & - & - & - & - & - & $+^{*}$ \\
\hline Tween 80 hydrolysis & + & - & - & - & - & - & - & - & - & - & - & - \\
\hline Aesculin hydrolysis & $+1-$ & - & - & - & $+1-$ & - & + & - & - & - & + & + \\
\hline Acetoin production & + & + & + & + & + & + & + & + & + & - & + & + \\
\hline \multicolumn{13}{|l|}{ Utilization as sole carbon source of: } \\
\hline Citrate & - & - & - & - & + & - & - & - & - & - & - & - \\
\hline D-Glucose & - & - & $+1-$ & $+1-$ & - & - & - & - & - & - & - & $+1-$ \\
\hline D-Ribose & $+1-$ & $+1-$ & $+1-$ & $+1-$ & $+1-$ & - & - & - & - & - & $+1-$ & $+1-$ \\
\hline Pyrazinamidase & + & + & + & + & + & + & + & - & - & + & + & + \\
\hline Pyrolidonyl arylamidase & + & - & + & + & + & - & + & - & - & - & + & + \\
\hline Urease & - & - & - & - & - & - & - & - & - & - & - & + \\
\hline Acid phosphatase & + & + & - & + & + & - & - & - & - & - & - & - \\
\hline Naphthol-AS-BI-phosphohydrolase & + & + & + & + & + & + & + & + & + & - & + & + \\
\hline Alkaline phosphatase & + & + & + & + & + & - & + & - & - & + & + & + \\
\hline$\alpha$-Glucosidase & + & $+1-$ & $+1-$ & + & - & - & - & - & - & - & + & + \\
\hline$\alpha$-Mannosidase & + & + & - & - & - & - & - & - & - & - & + & - \\
\hline
\end{tabular}

${ }^{*}$ After 2 weeks of incubation.

only one mismatch at nucleotide position 403 (Tortoli et al., 2004). In the present study, the proposed MAC species differed from the most closely related species by two positions in the 16S rRNA gene. We therefore completed genetic analyses by sequencing $h s p 65, r p o B$ and ITS-1.

Strain $4355387^{\mathrm{T}}$ shared $99.3 \%$ 16S rRNA gene sequence similarity, $51.8 \%$ ITS-1 similarity, $97.1 \%$ rpoB similarity and $97.8 \%$ hsp 65 similarity with the most closely related type strain, M. colombiense CIP $108962^{\mathrm{T}}$ (Tables 2 and 3). Strains 3256799 and $5351974^{\mathrm{T}}$ exhibited an identical sequence, which is closely related to that of strains 62863 and $5356591^{\mathrm{T}}$ and marginally related to the M. intracellulare/M. chimaera cluster. When this sequence was compared to that of M. chimaera DSM $44623^{\mathrm{T}}$, strains 3256799 and $5351974^{\mathrm{T}}$ exhibited sequence similarities of $99.6 \%$ for the $16 \mathrm{~S}$ rRNA gene, $97.8 \%$ for $r p o B, 98.0$ and $98.1 \%$ for $h s p 65$, and 90.1 and $90.4 \%$ for ITS-1, respectively. Strains 62863 and $5356591^{\mathrm{T}}$ exhibited sequence similarities of $99.8 \%$ for the 16S rRNA gene, 86.3 and $86.1 \%$ for ITS- 1 , 96.7 and $97.8 \%$ for $r p o B$, and 97.6 and $97.4 \%$ for $h s p 65$, respectively, with $M$. chimaera DSM $44623^{\mathrm{T}}$. In total, the $\geqslant 2$ mismatches in the $16 \mathrm{~S}$ rRNA gene sequence, the $>5$ mismatches in $r p o B$ and the $\geqslant 15$ mismatches in the ITS and $h$ sp65 sequences between strains $5356591^{\mathrm{T}}, 62863$, 3256799 and $5351974^{\mathrm{T}}$ and the closely related type strain M. chimaera DSM $44623^{\mathrm{T}}$, and between strain $4355387^{\mathrm{T}}$ and M. colombiense CIP $108962^{\mathrm{T}}$ were indicative of the existence of three novel species.

Furthermore, a phylogenetic tree derived from the 16S rRNA gene sequences demonstrated only $2 / 9$ nodes $(22 \%)$ with bootstrap values $\geqslant 96 \%$ (Fig. 1), giving poor confidence in this topology. The tree derived from the concatenated $16 \mathrm{~S}$ rRNA gene, rpoB, $h s p 65$ and ITS- 1 sequences (Devulder et al., 2005; Stackebrandt et al., 2002) yielded bootstrap values of $\geqslant 97 \%$ in $8 / 11$ nodes $(72 \%)$, indicating higher confidence in this representation (Fig. 2). This tree indicated that strain $4355387^{\mathrm{T}}$ was distinct from but closely related to M. colombiense CIP $108962^{\mathrm{T}}$. Furthermore, strains 3256799 and $5351974^{\mathrm{T}}$ formed a cluster that was distinct from but related to the cluster formed by strains 62863 and $5356591^{\mathrm{T}}$, and both clusters were marginally related to the $M$. intracellulare/M. chimaera cluster.

In this study, partial $r p o B$ sequencing identified five MAC isolates that could not be classified at the species level. 
Table 2. Sequence similarity (\%) for $r p o B$ (below the diagonal) and $h s p 65$ (above the diagonal) for MAC reference strains and the five atypical clinical isolates

Strains: 1, M. avium subsp. avium ATCC $25291^{\mathrm{T}} ; 2$, M. avium subsp. paratuberculosis ATCC $19698^{\mathrm{T}} ; 3$, M. avium subsp. silvaticum ATCC $49884^{\mathrm{T}}$; 4 , 'M. avium subsp. hominissuis' IWGMT 49; 5, M. chimaera DSM 44623 ${ }^{\mathrm{T}} ; 6$, M. intracellulare ATCC 15985; 7, M. colombiense CIP $108962^{\mathrm{T}}$; 8,62863 ( $r p o B$ and $h s p 65$ sequence accession nos are EF584444 and EU239788, respectively); 9, 5356591 ${ }^{\mathrm{T}}$ (EF584434 and EU239787); 10, 4355387 ${ }^{\mathrm{T}}$ (EF584445 and EU239790); 11, 3256799 (EF584435 and EU239791); 12, 5351974 ${ }^{\mathrm{T}}$ (rpoB sequence identical to EF584444; hsp65 sequence accession no. EU239792).

\begin{tabular}{|c|c|c|c|c|c|c|c|c|c|c|c|c|}
\hline \multirow[t]{2}{*}{ Strain } & \multicolumn{12}{|c|}{ Sequence similarity (\%) with: } \\
\hline & 1 & 2 & 3 & 4 & 5 & 6 & 7 & 8 & 9 & 10 & 11 & 12 \\
\hline 1. M. avium subsp. avium ATCC $25291^{\mathrm{T}}$ & & 99.0 & 100 & 95.7 & 94.7 & 94.8 & 95.0 & 94.3 & 94.2 & 95.7 & 94.8 & 94.7 \\
\hline 2. M. avium subsp. paratuberculosis ATCC $19698^{\mathrm{T}}$ & 99.7 & & 99.2 & 95.3 & 94.5 & 94.6 & 94.6 & 94.1 & 94.0 & 95.3 & 95.0 & 94.5 \\
\hline 3. M. avium subsp. silvaticum ATCC $49884^{\mathrm{T}}$ & 99.8 & 99.5 & & 95.7 & 94.7 & 94.8 & 95.0 & 94.3 & 94.2 & 95.7 & 94.8 & 94.7 \\
\hline 4. 'M. avium subsp. hominissuis' IWGMT 49 & 99.7 & 99.7 & 99.5 & & 91.0 & 91.1 & 91.5 & 90.9 & 90.8 & 92.0 & 91.1 & 91.0 \\
\hline 5. M. chimaera DSM $44623^{\mathrm{T}}$ & 94.7 & 94.7 & 94.6 & 94.9 & & 99.8 & 97.5 & 97.6 & 97.4 & 97.3 & 98.0 & 98.1 \\
\hline 6. M. intracellulare ATCC 15985 & 94.3 & 94.3 & 94.2 & 94.5 & 99.2 & & 97.6 & 97.6 & 97.5 & 97.0 & 98.1 & 98.2 \\
\hline 7. M. colombiense CIP $108962^{\mathrm{T}}$ & 92.9 & 92.9 & 92.8 & 92.9 & 93.9 & 93.5 & & 97.5 & 97.5 & 97.8 & 97.3 & 97.0 \\
\hline 8.62863 & 93.5 & 93.5 & 93.6 & 93.6 & 96.7 & 96.3 & 94.2 & & 99.8 & 96.7 & 98.0 & 97.2 \\
\hline 9. $5356591^{\mathrm{T}}$ & 94.6 & 94.6 & 94.7 & 94.7 & 97.8 & 97.4 & 93.3 & 98.8 & & 96.6 & 97.6 & 97.1 \\
\hline 10. $4355387^{\mathrm{T}}$ & 95.2 & 95.2 & 95.0 & 95.2 & 96.2 & 95.7 & 97.1 & 94.6 & 95.7 & & 97.4 & 96.9 \\
\hline 11. 3256799 & 94.6 & 94.6 & 94.5 & 94.7 & 97.8 & 97.4 & 93.9 & 97.1 & 98.1 & 96.3 & & 99.0 \\
\hline 12. $5351974^{\mathrm{T}}$ & 94.6 & 94.6 & 94.5 & 94.7 & 97.8 & 97.4 & 93.9 & 97.1 & 98.1 & 96.3 & 100 & \\
\hline
\end{tabular}

Phylogenetic analyses indicated that four of these five isolates were more closely related to the $M$. intracellulare/ $M$. chimaera group, although they belong to two different species that are distinct from both $M$. intracellulare and $M$. chimaera. The fifth isolate was more closely related to $M$. colombiense. Further polyphasic characterization of these five isolates indicated that these isolates were indeed representative of three novel MAC species. These data indicate that partial $r p o B$ sequencing was effective for the initial identification of MAC isolates. Moreover, the same PCR sequencing primer pair could be used to identify MAC isolates and rapidly growing mycobacteria, making $r p o B$ a convenient tool for initial investigations of Mycobacterium isolates (Adékambi et al., 2003). The data

Table 3. Sequence similarity (\%) for $16 \mathrm{~S}$ rRNA gene (below the diagonal) and ITS-1 (above the diagonal) sequences for MAC reference strains and the five atypical clinical isolates

Strains: 1, M. avium subsp. avium ATCC $25291^{\mathrm{T}} ; 2$, M. avium subsp. paratuberculosis ATCC $19698^{\mathrm{T}} ; 3$, M. avium subsp. silvaticum ATCC $49884^{\mathrm{T}}$; 4 , 'M. avium subsp. hominissuis' IWGMT 49; 5, M. chimaera DSM 44623 ${ }^{\mathrm{T}} ; 6$, M. intracellulare ATCC 15985; 7, M. colombiense CIP $108962^{\mathrm{T}}$; 8, 62863 (16S rRNA gene sequence identical to EU266632; ITS-1 sequence accession no. EU266630); 9, 5356591 ${ }^{\mathrm{T}}$ (16S rRNA gene and ITS-1 sequence accession nos EU266632 and EU266631, respectively); 10, 4355387 ${ }^{\mathrm{T}}$ (EF591053 and EF591051); 11, 3256799 (EF591054 and EF591050); 12, $5351974^{\mathrm{T}}$ (16S rRNA gene sequence identical to EF591054; ITS-1 sequence accession no. EU266628).

\begin{tabular}{|c|c|c|c|c|c|c|c|c|c|c|c|c|}
\hline \multirow[t]{2}{*}{ Strain } & \multicolumn{12}{|c|}{ Sequence similarity (\%) with: } \\
\hline & 1 & 2 & 3 & 4 & 5 & 6 & 7 & 8 & 9 & 10 & 11 & 12 \\
\hline 1. M. avium subsp. avium ATCC $25291^{\mathrm{T}}$ & & 100 & 100 & 99.7 & 45.4 & 44.2 & 62.5 & 35.5 & 35.5 & 43.3 & 38.8 & 38.3 \\
\hline 2. M. avium subsp. paratuberculosis ATCC $19698^{\mathrm{T}}$ & 100 & & 100 & 99.7 & 45.4 & 44.2 & 62.5 & 35.5 & 35.5 & 43.3 & 38.8 & 38.3 \\
\hline 3. M. avium subsp. silvaticum ATCC $49884^{\mathrm{T}}$ & 100 & 100 & & 99.7 & 45.4 & 44.2 & 62.5 & 35.5 & 35.5 & 43.3 & 38.8 & 38.3 \\
\hline 4. 'M. avium subsp. hominissuis' IWGMT 49 & 100 & 100 & 100 & & 45.4 & 44.5 & 62.2 & 35.7 & 35.7 & 43.3 & 39.0 & 38.5 \\
\hline 5. M. chimaera DSM $44623^{\mathrm{T}}$ & 96.3 & 96.3 & 96.3 & 96.3 & & 95.6 & 51.1 & 86.3 & 86.1 & 95.4 & 90.1 & 90.4 \\
\hline 6. M. intracellulare ATCC 15985 & 96.7 & 96.7 & 96.7 & 96.7 & 99.9 & & 50.6 & 87.4 & 87.2 & 95.6 & 90.8 & 90.6 \\
\hline 7. M. colombiense CIP $108962^{\mathrm{T}}$ & 96.7 & 96.7 & 96.7 & 96.7 & 99.4 & 99.4 & & 50.4 & 50.4 & 51.8 & 50.2 & 49.7 \\
\hline 8. 62863 & 96.4 & 96.4 & 96.4 & 96.4 & 99.8 & 99.5 & 99.5 & & 99.7 & 86.3 & 86.5 & 86.5 \\
\hline 9. $5356591^{\mathrm{T}}$ & 96.4 & 96.4 & 96.4 & 96.4 & 99.8 & 99.5 & 99.5 & 100 & & 86.1 & 86.3 & 86.3 \\
\hline 10. $4355387^{\mathrm{T}}$ & 96.6 & 96.6 & 96.6 & 96.6 & 99.9 & 99.9 & 99.3 & 99.5 & 99.5 & & 90.4 & 90.6 \\
\hline 11. 3256799 & 96.3 & 96.3 & 96.3 & 96.3 & 99.6 & 99.6 & 99.6 & 99.7 & 99.7 & 99.5 & & 99.3 \\
\hline 12. $5351974^{\mathrm{T}}$ & 96.3 & 96.3 & 96.3 & 96.3 & 99.6 & 99.6 & 99.6 & 99.7 & 99.7 & 99.5 & 100 & \\
\hline
\end{tabular}




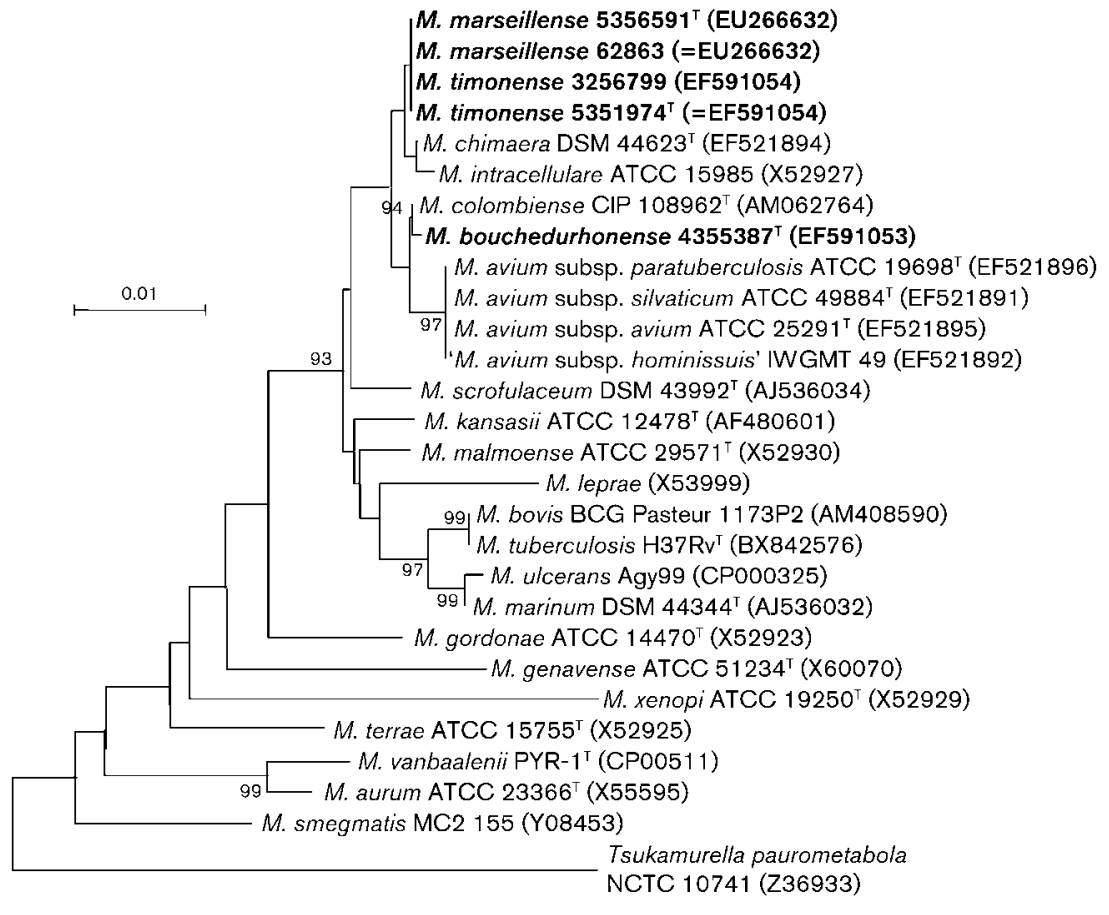

Fig. 1. $16 \mathrm{~S}$ rRNA gene sequence-based phylogenetic tree indicating the taxonomic relationships of the novel MAC species. Bootstrap support values $\geqslant 95 \%$, determined from 1000 replicates, are indicated at nodes. The sequence of Tsukamurella paurometabola NCTC 10741 was used as the outgroup. Bar, $1 \%$ difference in nucleotide sequence. presented here, in addition to the recent descriptions of $M$. chimaera (Tortoli et al., 2004) and M. colombiense (Murcia et al., 2006), suggest that the MAC consists of a heterogeneous group of mycobacteria that includes many novel species. More detailed phylogenetic and taxonomic descriptions of MAC species are warranted in order to provide a solid support from which to investigate MAC infections in animals and humans. Such descriptions could form the basis for analysis of mycobacterial sources and routes of transmission.

\section{Description of Mycobacterium marseillense sp. nov.}

Mycobacterium marseillense (mar.seil.len'se. N.L. neut. adj. marseillense pertaining to Marseille, where the first strains were isolated).
Cells are small $(0.92-1.03 \times 0.50-0.54 \mu \mathrm{m})$, acid-fast, Gram-positive bacilli. Colonies are non-pigmented and grow on $5 \%$ sheep blood agar, Middlebrook 7H10 agar and egg-based Löwenstein-Jensen slants after 10 days incubation at $30{ }^{\circ} \mathrm{C}$. Exhibits a unique morphology with pseudo-mycelium formation. Hydrolyses Tween 80, but does not reduce potassium tellurite. It shares $96.4-99.8 \%$ 16S rRNA gene and 93.3-97.8\% rpoB, 94.0-97.5\% hsp65 and 35.5-87.2\% ITS-1 sequence similarities with MAC reference strains $M$. avium ATCC $25291^{\mathrm{T}}$, M. intracellulare ATCC 15985, M. chimaera DSM $44623^{\mathrm{T}}$ and M. colombiense CIP $108962^{\mathrm{T}}$.

The type strain is $5356591^{\mathrm{T}}\left(=\mathrm{CIP} 109828^{\mathrm{T}}=\mathrm{CCUG}\right.$ $56325^{\mathrm{T}}=\mathrm{CSUR} \mathrm{P} 30^{\mathrm{T}}$ ), sequevar MAC-X1, which was recovered from sputum. Strain 62863 (=CCUG 56324

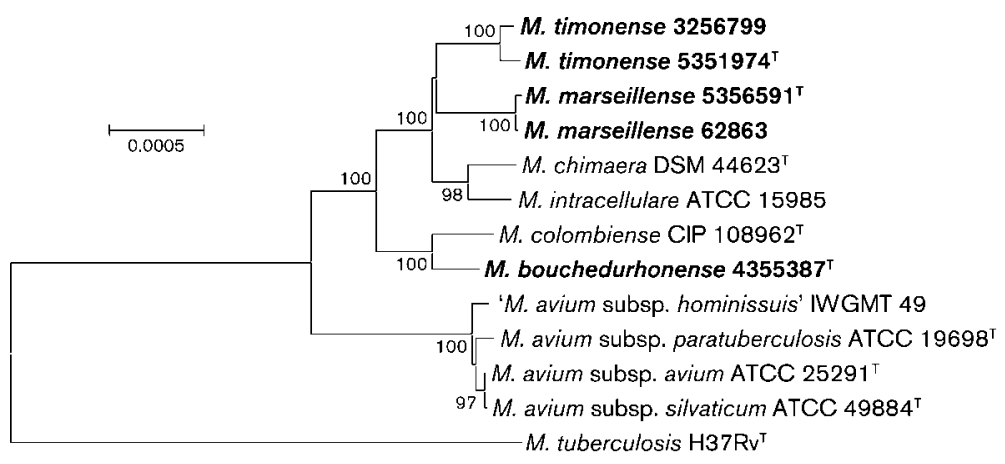

Fig. 2. Phylogenetic tree based on concatenated 16S rRNA gene, ITS-1, rpoB and hsp65 sequences, showing relationships between the clinical isolates $62863,5351974^{\top}, 3256799$, $5356591^{\top}$ and $4355387^{\top}$ and strains of the four MAC reference species using the neighbour-joining method and Kimura's two-parameter distance correction model. Bootstrap support values $\geqslant 95 \%$, determined from 1000 replicates, are indicated at nodes. Mycobacterium tuberculosis $\mathrm{H}_{37 \mathrm{Rv}^{\top}}$ was used as the outgroup. Bar, $0.05 \%$ difference in nucleotide sequence. 
$=\mathrm{CIP} 109832=\mathrm{CSUR}$ P31), sequevar MAC-D, is a reference strain.

\section{Description of Mycobacterium timonense sp. nov.}

Mycobacterium timonense (ti.mo.nen'se. N.L. neut. adj. timonense pertaining to La Timone, the name of a hospital in Marseille, France, where the first strains were isolated).

Cells are acid-fast, Gram-positive bacilli with a mean size of $0.9 \times 0.45 \mu \mathrm{m}$. Unpigmented colonies grow on $5 \%$ sheep blood agar, Middlebrook $7 \mathrm{H} 10$ agar and egg-based Löwenstein-Jensen slants after 10 days incubation at $30{ }^{\circ} \mathrm{C}$. Positive for acid phosphatase activity. It shares 96.3-99.6\% 16S rRNA gene, 96.3-99.6\% rpoB, 91.098.2\% hsp65 and 38.3-90.6\% ITS-1 sequence similarities with MAC reference strains.

The type strain is $5351974^{\mathrm{T}}\left(=\mathrm{CIP} 109830^{\mathrm{T}}=\mathrm{CCUG} 56329^{\mathrm{T}}\right.$ $=$ CSUR P $32^{\mathrm{T}}$ ), sequevar MAC-K, which was recovered from sputum. Strain 3256799 (=CCUG 56328 =CIP 109829 =CSUR P33), sequevar MAC-L, is a reference strain.

\section{Description of Mycobacterium bouchedurhonense sp. nov.}

Mycobacterium bouchedurhonense (bou.che.du.rho.nen'se. N.L. neut. adj. bouchedurhonense pertaining to Bouches du Rhône, the name of the département around Marseille, France, where the type strain was recovered).

Cells are small $(0.87 \times 0.49 \mu \mathrm{m})$, acid-fast, Gram-positive bacilli. Colonies are non-pigmented and grow on $5 \%$ sheep blood agar, Middlebrook 7H10 agar and egg-based Löwenstein-Jensen slants after 10 days incubation at $30{ }^{\circ} \mathrm{C}$. Differs from MAC species in its ability to use citrate as a carbon source. It shares 96.6-99.9\% 16S rRNA gene, 95.097.1\% rpoB, 43.3-51.8\% hsp65 and 92.0-97.8\% ITS-1 sequence similarities with MAC reference strains.

The type strain is $4355387^{\mathrm{T}}\left(=\mathrm{CIP} 109827^{\mathrm{T}}=\mathrm{CCUG}\right.$ $56331^{\mathrm{T}}=$ CSUR P $34^{\mathrm{T}}$ ), sequevar MAC-X2, which was recovered from sputum.

\section{References}

Adékambi, T., Colson, P. \& Drancourt, M. (2003). rpoB-based identification of nonpigmented and late-pigmenting rapidly growing mycobacteria. J Clin Microbiol 41, 5699-5708.

Ben Salah, I., Adékambi, T., Raoult, D. \& Drancourt, M. (2008). $r p o B$ sequence-based identification of Mycobacterium avium complex species. Microbiology 154, 3715-3723.

Devulder, G., Pérouse de Montclos, M. \& Flandrois, J. P. (2005). A multigene approach to phylogenetic analysis using the genus Mycobacterium as a model. Int J Syst Evol Microbiol 55, 293-302.

Djelouagji, Z. \& Drancourt, M. (2006). Inactivation of cultured Mycobacterium tuberculosis organisms prior to DNA extraction. J Clin Microbiol 44, 1594-1595.
Frothingham, R. \& Wilson, K. H. (1993). Sequence-based differentiation of strains in the Mycobacterium avium complex. J Bacteriol 175, 2818-2825.

Kent, P. T. \& Kubica, G. P. (1985). Public Health Mycobacteriology: a Guide for the Level III Laboratory. Atlanta, GA: US Department of Health and Human Services, Centers for Disease Control.

Kumar, S., Tamura, K. \& Nei, M. (2004). MEGA3: integrated software for molecular evolutionary genetics analysis and sequence alignment. Brief Bioinform 5, 150-163.

Lebrun, L., Weill, F. X., Lafendi, L., Houriez, F., Casanova, F., Gutierrez, M. C., Ingrand, D., Lagrange, P., Vincent, V. \& Herrmann, J. L. (2005). Use of the INNO-LiPA-MYCOBACTERIA assay (version 2) for identification of Mycobacterium avium-Mycobacterium intracellulare-Mycobacterium scrofulaceum complex isolates. J Clin Microbiol 43, 2567-2574.

Mignard, S. \& Flandrois, J. P. (2008). A seven-gene, multilocus, genus-wide approach to the phylogeny of mycobacteria using supertrees. Int J Syst Evol Microbiol 58, 1432-1441.

Mijs, W., de Haas, P., Rossau, R., Van der Laan, T., Rigouts, L., Portaels, F. \& van Soolingen, D. (2002). Molecular evidence to support a proposal to reserve the designation Mycobacterium avium subsp. avium for bird-type isolates and ' $M$. avium subsp. hominissuis' for the human/porcine type of M. avium. Int J Syst Evol Microbiol 52, 1505-1518.

Murcia, M. I., Tortoli, E., Menendez, M. C., Palenque, E. \& Garcia, M. J. (2006). Mycobacterium colombiense sp. nov., a novel member of the Mycobacterium avium complex and description of MAC-X as a new ITS genetic variant. Int J Syst Evol Microbiol 56, 20492054.

Pfyffer, G. E. (2007). Mycobacterium: general characteristics, laboratory detection, and staining procedures. In Manual of Clinical Microbiology, 9th edn, pp. 543-572. Edited by P. R. Murray, E. J. Baron, J. H. Jorgensen, M. L. Landry \& M. A. Pfaller. Washington, DC: American Society for Microbiology.

Stackebrandt, E., Frederiksen, W., Garrity, G. M., Grimont, P. A. D., Kämpfer, P., Maiden, M. C. J., Nesme, X., Rosselló-Mora, R., Swings, J. \& other authors (2002). Report of the ad hoc committee for the re-evaluation of the species definition in bacteriology. Int $J$ Syst Evol Microbiol 52, 1043-1047.

Thompson, J. D., Gibson, T. J., Plewniak, F., Jeanmougin, F. \& Higgins, D. G. (1997). The CLUSTAL_X windows interface: flexible strategies for multiple sequence alignment aided by quality analysis tools. Nucleic Acids Res 25, 4876-4882.

Tortoli, E., Rindi, L., Garcia, M. J., Chiaradonna, P., Dei, R., Garzelli, C., Kroppenstedt, R. M., Lari, N., Mattei, R. \& other authors (2004). Proposal to elevate the genetic variant MAC-A, included in the Mycobacterium avium complex, to species rank as Mycobacterium chimaera sp. nov. Int J Syst Evol Microbiol 54, 1277-1285.

Turenne, C. Y., Semret, M., Cousins, D. V., Collins, D. M. \& Behr, M. A. (2006). Sequencing of $h s p 65$ distinguishes among subsets of the Mycobacterium avium complex. J Clin Microbiol 44, 433-440.

Vincent, V., Brown-Elliott, B. A., Jost, K. C., Jr \& Wallace, R. J., Jr (2003). Mycobacterium: phenotypic and genotypic identification. In Manual of Clinical Microbiology, 8th edn, pp. 560-584. Edited by P. R. Murray, E. J. Baron, J. H. Jorgensen, M. A. Pfaller \& R. H. Yolken. Washington, DC: American Society for Microbiology.

Weisburg, W. G., Barns, S. M., Pelletier, D. A. \& Lane, D. J. (1991). $16 \mathrm{~S}$ ribosomal DNA amplification for phylogenetic study. J Bacteriol 173, 697-703. 